\title{
The game of End-Nim
}

\author{
Michael H. Albert \\ Dept. of Computer Science \\ Otago University \\ Dunedin, New Zealand \\ malbert@atlas.otago.ac.nz
}

\author{
Richard J. Nowakowski * \\ Dept. of Mathematics \& Statistics \\ Dalhousie University, \\ Halifax, N.S. Canada B3J 3H5 \\ rjn@mathstat.dal.ca
}

\begin{abstract}
In the game of End-Nim two players take turns in removing one or more boxes from a string of non-empty stacks. At each move boxes may only be taken from the two stacks which form the ends of the string (unless only one stack remains!). We give a solution for both impartial and partizan versions of the game and explain the significance of the mystic hieroglyphs:
\end{abstract}

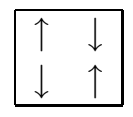

AMS subject classifications: 91A46 (primary), 05B99 (secondary).

Submitted Aug 20 2000, Accepted Feb 62001.

\section{Introduction}

Lorraine and Roger are fork-lift operators, with a penchant for combinatorial games. Many of the warehouses from which they need to remove boxes have the boxes in stacks, with the stacks arranged in a row. Only boxes belonging to the stacks at the end of a row are accessible, but the fork-lifts are sufficiently powerful that they can move an entire stack of boxes if necessary. The game which Lorraine and Roger play most often is won by the player who removes the last box from a row of stacks. Usually they play fair and allow each other to remove boxes from either end. Sometimes, in particularly narrow warehouses each of them is assigned a specific end to work from.

We have dubbed the game which they play End-Nim, and the two versions are of course the impartial and partizan versions of the game. Formally an End-Nim position is a sequence of positive integers, and the legal moves in the impartial version are to reduce

\footnotetext{
*Partially supported by a grant from NSERC and the Beverley Trust of the Department of Mathematics and Statistics at Otago University.
} 
the first or last element of the sequence by at least one, or to delete it entirely. In the partizan version one player is restricted to work at the head of the sequence, and the other at the tail.

The motivation for considering these questions comes from a 1990 conversation between A. Fraenkel and J. H. Conway. Fraenkel asked Conway about the impartial version of the game and during the day they worked out a solution. Unfortunately this solution was lost and neither of the principals have been able to recall what it was. We hope to reveal all. The impartial version (under the name "Burning-the-candle-at-both-ends") is also discussed (with some generalizations) as problem number 23 in R. Guy's list of unsolved problems [2].

We use boldface latin characters to stand for strings of positive integers, and non-bold characters for single positive integers. Concatenation of strings is denoted by juxtaposition, and repetition by exponentiation. The length of a string is simply the number of characters in that string.

\section{Impartial End-Nim}

Lorraine seems to be much better at this game than Roger, and one day he notices that while they are playing Lorraine is glancing occasionally at a scrap of paper taped to her steering wheel. At lunchtime, after another defeat, he sneaks a peek at the paper. What he sees is:

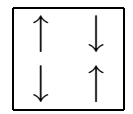

In the remainder of this section we will try to explain the significance of this mysterious notation.

Let us establish at the outset that we do not intend to compute the Nim-values of general End-Nim positions. As partial evidence of the "chaotic" nature of these values we present the following table of Nim-values for the positions $a 4 b$, for $1 \leq a \leq 16$ and 
$1 \leq b \leq 16$

\begin{tabular}{r||c|cc|cccc|cccccccc|c} 
& $\mathbf{1}$ & $\mathbf{2}$ & $\mathbf{3}$ & $\mathbf{4}$ & $\mathbf{5}$ & $\mathbf{6}$ & $\mathbf{7}$ & $\mathbf{8}$ & $\mathbf{9}$ & $\mathbf{1 0}$ & $\mathbf{1 1}$ & $\mathbf{1 2}$ & $\mathbf{1 3}$ & $\mathbf{1 4}$ & $\mathbf{1 5}$ & $\mathbf{1 6}$ \\
\hline \hline $\mathbf{1}$ & 0 & 1 & 2 & 3 & 4 & 6 & 7 & 8 & 9 & 10 & 11 & 12 & 13 & 14 & 15 & 16 \\
\hline $\mathbf{2}$ & 1 & 0 & 3 & 2 & 5 & 4 & 8 & 7 & 10 & 9 & 12 & 11 & 14 & 13 & 16 & 15 \\
$\mathbf{3}$ & 2 & 3 & 0 & 1 & 6 & 5 & 4 & 9 & 8 & 11 & 10 & 13 & 12 & 15 & 14 & 17 \\
\hline $\mathbf{4}$ & 3 & 2 & 1 & 4 & 7 & 8 & 5 & 6 & 11 & 12 & 9 & 10 & 15 & 16 & 13 & 14 \\
$\mathbf{5}$ & 4 & 5 & 6 & 7 & 0 & 3 & 2 & 10 & 12 & 8 & 13 & 9 & 11 & 17 & 18 & 19 \\
$\mathbf{6}$ & 6 & 4 & 5 & 8 & 3 & 0 & 1 & 11 & 7 & 13 & 14 & 15 & 10 & 9 & 12 & 18 \\
$\mathbf{7}$ & 7 & 8 & 4 & 5 & 2 & 1 & 0 & 13 & 6 & 15 & 16 & 14 & 17 & 11 & 9 & 10 \\
\hline $\mathbf{8}$ & 8 & 7 & 9 & 6 & 10 & 11 & 13 & 0 & 1 & 2 & 3 & 4 & 5 & 18 & 17 & 21 \\
$\mathbf{9}$ & 9 & 10 & 8 & 11 & 12 & 7 & 6 & 1 & 0 & 3 & 2 & 5 & 4 & 19 & 20 & 22 \\
$\mathbf{1 0}$ & 10 & 9 & 11 & 12 & 8 & 13 & 15 & 2 & 3 & 0 & 1 & 6 & 7 & 4 & 5 & 23 \\
$\mathbf{1 1}$ & 11 & 12 & 10 & 9 & 13 & 14 & 16 & 3 & 2 & 1 & 0 & 7 & 6 & 5 & 4 & 8 \\
$\mathbf{1 2}$ & 12 & 11 & 13 & 10 & 9 & 15 & 14 & 4 & 5 & 6 & 7 & 0 & 1 & 2 & 3 & 24 \\
$\mathbf{1 3}$ & 13 & 14 & 12 & 15 & 11 & 10 & 17 & 5 & 4 & 7 & 6 & 1 & 0 & 3 & 2 & 25 \\
$\mathbf{1 4}$ & 14 & 13 & 15 & 16 & 17 & 9 & 11 & 18 & 19 & 4 & 5 & 2 & 3 & 0 & 1 & 6 \\
$\mathbf{1 5}$ & 15 & 16 & 14 & 13 & 18 & 12 & 9 & 17 & 20 & 5 & 4 & 3 & 2 & 1 & 0 & 7 \\
\hline $\mathbf{1 6}$ & 16 & 15 & 17 & 14 & 19 & 18 & 10 & 21 & 22 & 23 & 8 & 24 & 25 & 6 & 7 & 0
\end{tabular}

Note the intrusions of values equal to $2^{k}$ or larger within the blocks corresponding to positions with all the heaps of size smaller than $2^{k}$. Also, within each row (or column) the sequence appears to be ultimately arithmetico-periodic. This we have proved for the first few values of $a$. The argument, not presented here, is not terribly illuminating. The amount of noise before the beginning of the regular behavior is variable, and the ultimate periods (being of lengths a power of two) themselves double in length occasionally.

Instead of evaluating End-Nim positions, we will aim only to provide an easy algorithm for recognizing the outcome classes, i.e. $\mathcal{P}$ (previous player win) positions and $\mathcal{N}$ (next player win) positions. Of course this is all that is really necessary so long as you wish to play End-Nim in isolation.

The first basic observation is the following:

Proposition 1 If $x \mathbf{w} y \in \mathcal{P}$ with both $x$ and $y$ greater than 0 , then $|x-y| \leq 1$.

Proof: Since $x \mathbf{w} y \in \mathcal{P}$, then for all $1 \leq i<y, x \mathbf{w} i \in \mathcal{N}$. Consider such a position $x \mathbf{w} i$. The first player's winning move cannot be on the right hand side, since all of the positions $x \mathbf{w} j$ for $0 \leq j<y$ are in $\mathcal{N}$. Thus any winning first move must be on the left hand side. For each $i$, choose $x_{i}$ so that $x_{i} \mathbf{w} i$ is in $\mathcal{P}$.

If $j<k$ then $x_{j} \neq x_{k}$ for otherwise $x_{k} \mathbf{w} k$ would have $x_{k} \mathbf{w} j$ as a $\mathcal{P}$-option, implying that $x_{k} \mathbf{w} k \in \mathcal{N}$ contrary to the choice of $x_{k}$. So the $x_{i}$ are all distinct. As there are only $x$ possible values for the $x_{i}$ it must be the case that $x \geq y-1$.

By symmetry, $y \geq x-1$. Thus $|x-y| \leq 1$.

The proposition above already means that from any position the number of potential good moves (i.e. those which might lead to $\mathcal{P}$ positions) is at most four, and greatly simplifies the considerations of strategy for either player. 
In what follows we will be representing an (almost) arbitrary string in the form:

$$
x^{n} a \mathbf{w} b y^{m}
$$

with the following understandings: $n, m \geq 1, x \neq a, b \neq y$, w might be empty, and $a$ and $b$ might be the same character. In fact, simply set $a$ to equal the value of the first character of the string which is not equal to the head, and $b$ the value of the last character of the string not equal to its tail. The only non-empty strings which cannot be so represented are ones of one of the two forms:

$$
x^{n} \quad \text { or } \quad x^{n} y^{m}
$$

For strings of the first type, with $n$ even, the second player wins by copying his opponent's move but on the other side of the of the string so these positions are in $\mathcal{P}$. Of course if $n$ is odd, these positions are in $\mathcal{N}$ since the first player can win by deleting one of the stacks and then following the strategy prescribed above. For strings of the second type we'll pretend that $a=y$ and $b=x$ but $n$ and $m$ are not to be changed. For example, in 22244444, $x=2, y=4, n=3, m=5, a=4$ and $b=2$.

Our Most Assiduous Reader, Omar [1] p42, will have noticed that in determining whether or not a position is in $\mathcal{P}$, the parity of $n$ and $m$ is important, as well as the relationship between $x$ and $y$, and the direction of the transitions from $x$ to $a$ and $y$ to $b$. We can be a little more precise as the theorem which follows will establish that this information alone is sufficient to determine whether or not a position is in $\mathcal{P}$.

In order to state that theorem though we need to explain the interpretation of the symbols on Lorraine's steering wheel. In fact these symbols encapsulate a description of all the non-trivial $\mathcal{P}$ positions. Namely, suppose we are given a string $x^{n} a \mathbf{w} b y^{m}$ which might be in $\mathcal{P}$ (so at the very least $|x-y| \leq 1$ ). Each end of the string has a parity (denoted, a little unusually, 1 or 2) and a direction associated with it.

- For the left end of the string, the parity of $n$ (recorded as 1 for odd, and 2 for even), and an $\uparrow$ if $x<a$ or a $\downarrow$ if $x>a$.

- For the right end, the parity of $m$ and an $\uparrow$ if $y<b$ or a $\downarrow$ if $y>b$.

Now think of 1 and 2 as standing for "first" and "second" column in Lorraine's diagram. The rules above determine a cell in the diagram for the left end and one for the right end (possibly the same cell). The position is in $\mathcal{P}$ precisely when the order relationship between $x$ and $y$ (the same as, above, or below) is the same as the relationship of the height of the corresponding cells. For example, in 33323433, $x=3, n=3, a=2$ so $x>a$ and the corresponding cell in the diagram is that in the first column which contains $\downarrow$ marked by $*$ in

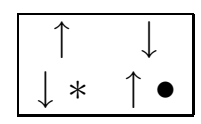

Also, $y=3, m=2$ and $b=4$ so $y<b$ and the corresponding cell is that in the second column which contains $\uparrow$. This is indicated by $\bullet$. The position is a $\mathcal{P}$ position because $x=y$ and the corresponding cells are on the same level. 
Theorem 2 The $\mathcal{P}$ positions of End-Nim are precisely the ones described above, the positions $x^{n}$ for $n$ even, and the empty position.

Proof: It suffices to show that any move from such a position takes us outside the class stated in the theorem, and that from anywhere outside the class we can move to a position in the class. The first part is quite easy, and we omit it.

Suppose first that we have a position where $y>x+1$. Here and elsewhere we will repeatedly make use of the symmetry of the game under reversal of the original string, so this argument also deals with the case where $y<x-1$. If $m>1$ then by reducing $y$ to $x$ or $x+1$ we achieve $1 \uparrow$ at the right hand end. If the left hand end is "top row" choose the $x$ option, if it's "bottom row" choose the $x+1$ option. We can choose similarly if $m=1$, but $|b-x|>1$.

If $m=1$ and $|b-x| \leq 1$ there are several cases to consider. Suppose that the $x$ end is "top row". If $b=x+1$ we achieve $1 \uparrow$ at the right, by reducing $y$ to $x$. If $b=x$ our options of either reducing $y$ to $x$ or deleting it entirely, allow us to achieve an even or an odd number of $x$ 's at the tail of the string, and we choose whichever one is appropriate to the new transition. If $b=x-1$ then by reducing $y$ to $x-1$ or deleting it entirely, we achieve a suitable bottom row position.

If the $x$-end was bottom row then reducing $y$ to $x+1$ achieves $1 \uparrow$ in all cases except when there is a singleton $y$, and the $y$-end is $1 \downarrow$. In this case, if $b=x$ or $x+1$ then reducing $y$ to $b$ or deleting it entirely will achieve an appropriate cell. If $b=x-1$ then reducing $y$ to $x$ achieves $1 \downarrow$.

We conclude that if a position is patently $\mathcal{N}$ (because the difference between the ends is too big) then we can in one move produce a position of one of the types which we claim are in $\mathcal{P}$.

Now we consider positions where $|x-y| \leq 1$ but the "level" of the ends is not appropriate. In each case we argue that there is a move which "works", that is, it produces a position in the list which we claim to be $\mathcal{P}$. The analysis is somewhat similar to what was done above, so we present it in the form of a table, dealing with five exceptional cases (numbered with superscripts) subsequently. Entries marked with a dash indicate cases which are in $\mathcal{P}$. We assume throughout that $y \geq x$. A notation of 0 means "delete the heap entirely". The values in parentheses denote the cell type achieved at the $y$-end by the indicated move. The columns are labeled by the type of the $y$-end, and its value, the rows simply by the type of the $x$-end.

\begin{tabular}{c|cc|cc|ccc|cc|}
\multicolumn{1}{c}{$1 \uparrow$} & \multicolumn{2}{c}{$1 \downarrow$} & \multicolumn{2}{c}{$2 \uparrow$} & \multicolumn{2}{c}{$2 \downarrow$} \\
& $x+1$ & $x$ & $x+1$ & $x$ & $x+1$ & $x$ & $x+1$ & $x$ \\
\hline $1 \uparrow$ & $x(1 \uparrow)$ & - & $x(1 \uparrow)$ & $0^{1}(2 \downarrow)$ & $x(1 \uparrow)$ & $0(1 \uparrow)$ & $x(1 \uparrow)$ & - \\
$1 \downarrow$ & - & $0^{2}(2 \uparrow)$ & $x(1 \downarrow)$ & - & $0(1 \uparrow)$ & - & - & $0(1 \downarrow)$ \\
$2 \uparrow$ & - & $*^{3}(1 \uparrow)$ & $0^{4}(2 \downarrow)$ & - & $0(1 \uparrow)$ & - & - & $0(1 \downarrow)$ \\
$2 \downarrow$ & $x(1 \uparrow)$ & - & $x(1 \uparrow)$ & $0^{5}(2 \downarrow)$ & $x(1 \uparrow)$ & $0(1 \uparrow)$ & $x(1 \uparrow)$ & - \\
\hline
\end{tabular}

\section{Exceptions:}

1. The $y$ end is $b x$ with $b<x$. If $b<x-1$ then reduce $y$ to $x-1$ achieving $1 \downarrow$. If $b=x-1$ then reducing $y$ to one of $x-1$ or 0 will achieve a bottom row position. 
2. This is the reverse of the above case.

3. This is the reverse of the case $(1 \uparrow, 2 \uparrow)$, a heap must be removed from the even end to produce a $(1 \uparrow, 1 \uparrow)$ position

4. The $y$-end is $b(x+1)$ with $b<x+1$. If $b<x$ then reducing $y$ to $x$ gives $1 \downarrow$. If $b=x$ then reducing $y$ to either $x$ or 0 will achieve a bottom row position.

5. This is the reverse of the case $(1 \downarrow, 2 \uparrow)$.

That concludes the proof that the positions we have described are in fact all the $\mathcal{P}$ positions, and thereby vindicates Lorraine's strategy.

\section{Partizan End-Nim}

In the partizan form of End-Nim the two players Lorraine and Roger are restricted to remove boxes from only the leftmost, or the rightmost stacks respectively. We should mention that these two always wear their softball jackets. Hers has a big L on the back, his has a big $\mathrm{R}$. To agree with the usual nomenclature of [1] we will refer to the players as Left and Right. For example, Left's legal options from $(2)(3)(1)$ are to $(1)(3)(1)$, or (3)(1), while Right has only one legal move, to (2)(3).

We do not intend to consider the exact values of partizan End-Nim positions in the sense of [1], but like the impartial version, only to determine the outcome classes. In the partizan theory of combinatorial games there are four outcome classes:
$\mathcal{N}$ the first player can force a win;
$\mathcal{P}$ the second player can force a win;
$\mathcal{L} \quad$ Left can force a win regardless of who moves first; and
$\mathcal{R}$ Right can force a win regardless of who moves first.

When unsure of the precise nature of a position we may write something like: $\mathbf{w} \in\{\mathcal{L}, \mathcal{R}\}$ to indicate that $\mathbf{w}$ is known or supposed to be a $\mathcal{L}$ or $\mathcal{R}$ position. In many cases we will state results relating specifically to Left or to Right. It is understood that corresponding results apply symmetrically to the other player.

Our first lemma simplifies the analysis of this game enormously since it implies that to analyze any given position we need only consider two different moves from that position for each player.

Lemma 3 If Right has a winning move from $\mathbf{w} x$, then one of:

- removing a single box,

- removing the entire stack,

is a winning move. 
Proof: If $x=1$ or removing the whole stack is a winning move then there is nothing to prove (in particular this applies if $\mathbf{w}$ is empty). So suppose that some move to $\mathbf{w} y$ $(y>0)$ is winning for Right. His options after Left's next move are a subset of what his options would have been had he simply removed one box from the stack. Thus whatever option wins for him, would also win if his first move were to $\mathbf{w}(x-1)$.

If the rightmost stack is sufficiently large, then the position will be a win for Right. Specifically there is a threshold function $R$ from non-empty strings of positive integers to positive integers such that $R(\mathbf{w})$ is the least positive integer $y$ for which $\mathbf{w} y \in \mathcal{R}$. We note in passing that $R(\mathbf{w}) \leq\left(\sum \mathbf{w}\right)+1$, and that for all $y \geq R(\mathbf{w}), \mathbf{w} y \in \mathcal{R}$. There is a corresponding threshold function $L$, on the left. The $\mathcal{L}$-threshold of a string is just the $\mathcal{R}$-threshold of its reversal. We invite Omar and the rest of our readers to attempt to compute the left and right thresholds of the strings 35451 and 35551.

More generally we may consider the sequence of types of the positions w $i$ for $i \geq 1$ (we think of this as the right phase diagram of w.)

Proposition 4 The right phase diagram of $\mathbf{w}$ consists either of:

- a string (possibly empty) of $\mathcal{N}$ 's followed by $\mathcal{R}$ 's, or,

- a string of $\mathcal{L}$ 's (again possibly empty) followed by a single $\mathcal{P}$, and then $\mathcal{R}$ 's.

Proof: Let $y=R(\mathbf{w})-1$ and suppose that $y>0$ (else there is nothing to prove). Since $\mathbf{w}(y+1)$ is in $\mathcal{R}$ it must be the case that either $\mathbf{w}$ or $\mathbf{w} y$ is a win for Right if Left moves first.

In the former case, we have that $\mathbf{w} \in\{\mathcal{P}, \mathcal{R}\}$. Then Right has a winning first move from $\mathbf{w} i$ for $1 \leq i \leq y$ so these positions are all in $\mathcal{N}$.

In the latter case (where $\mathbf{w} y$ is win for Right moving second), we must have that $\mathbf{w} y \in \mathcal{P}$ (since $y<R(\mathbf{w})$ we know the position is not in $\mathcal{R}$ ). Then all the positions $\mathbf{w} i$ for $1 \leq i<y$ must be in $\mathcal{N}$ or $\mathcal{L}$ since these positions are right options of $\mathbf{w} y$ and so Left wins them as first player. However none can be in $\mathcal{N}$ since Right's good first move from such a position would have been available as an option from $\mathbf{w} y$.

Given a $\mathcal{P}$ position, the following lemma allows us to construct a whole family of them:

Lemma 5 If $a \mathbf{w} b \in \mathcal{P}$ then $(a+1) \mathbf{w}(b+1) \in \mathcal{P}$.

Proof: Clearly it suffices by symmetry to establish that Right has no win as first player from the second position. If he did then either $(a+1) \mathbf{w} b$ or $(a+1) \mathbf{w}$ would be winning for him. The former is easily countered by Left moving to the known $\mathcal{P}$ position, while the latter is countered by Left's winning option from $a w$.

Given a string $\mathbf{w}$ we can consider the full phase diagram of $\mathbf{w}$ which consists of the types of all the strings of the form $a \mathbf{w} b$ where $a, b>0$. We write this diagram as a quarter infinite array, with the value of $a$ increasing upwards, and the value of $b$ increasing as we move to the right ${ }^{1}$. As a consequence of Proposition 4 (applied at both ends) and the

\footnotetext{
${ }^{1}$ Left makes it hotter while Right applies the pressure!
} 
lemma above we see that wherever we find a $\mathcal{P}$ in the phase diagram, there is a diagonal row of $\mathcal{P}$ 's extending upwards from it, all entries to the left or above such a $\mathcal{P}$ are $\mathcal{L}$ 's, and all to the right or below are $\mathcal{R}$ 's.

If there is a $\mathcal{P}$ in the first row or first column then the phase diagram consists entirely of $\mathcal{P}$ 's, $\mathcal{L}$ 's, and $\mathcal{R}$ 's. If not, then there is a leftmost bottommost $\mathcal{P}$ (outside of the first row and column) and the entries in the part of the array below and to the left of it are all $\mathcal{N}$ 's, while the entries in the remainder of the table are $\mathcal{R}$ 's, $\mathcal{L}$ 's, and $\mathcal{P}$ 's. This particular "leftmost bottommost" $\mathcal{P}$ will be called the triple point of $\mathbf{w}$. In fact, we'll abuse further our rather fanciful terminology and call the leftmost-bottommost $\mathcal{P}$ in any phase diagram its triple point. Note that the triple point must exist since, by Proposition 4 , the $\mathcal{N}$ region forms a rectangle bounded on top by $\mathcal{L}$ s and on the right by $\mathcal{R}$ s. The intersection of the $\mathcal{L}$ row and $\mathcal{R}$ column can only be a $\mathcal{P}$.

The key thing to realize is that simply knowing the position of the triple point of any phase diagram determines the phase diagram completely. An example of a phase diagram may prove helpful. Consider $\mathbf{w}=241$. Then it is easy to see that $\mathbf{w} \in \mathcal{N}$, and so $1 \mathbf{w} 1 \in \mathcal{N}$ also. Moreover $5241 \in \mathcal{P}$, and $2416 \in \mathcal{P}$, so $L(w)=6$ and $R(w)=7$. Corollary 7 to follow then establishes that the triple point is $6 \mathbf{w} 7$, and so the phase diagram is as shown below.

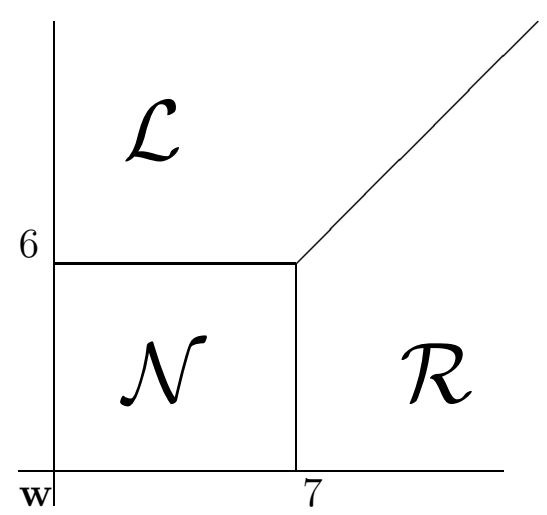

Note that the vertical and horizontal lines only serve to separate the $\mathcal{N}$ region from the $\mathcal{L}$ and $\mathcal{R}$ regions. Only the points on the diagonal line beginning from $6 \mathrm{w} 7$ represent $\mathcal{P}$ positions.

The observation that any $a \mathbf{w} b \in \mathcal{N}, a, b>1$ implies $1 \mathrm{w} 1 \in \mathcal{N}$ plus the observation above that the $1 \mathbf{w} 1$ entry of the phase diagram is the same as the type of $\mathbf{w}$ (since the play from $1 \mathrm{w} 1$ is forced for the first two moves) implies the following remarkable:

Corollary 6 There are no $\mathcal{N}$ positions of even length.

Proof: There are no $\mathcal{N}$ positions of length 2 .

Somewhat less surprisingly:

Corollary 7 If $\mathbf{w}$ has odd length then its triple point is $L(\mathbf{w}) \mathbf{w} R(\mathbf{w})$. 
Proof: Let $\mathbf{w}$ have odd length. First we check that $L(\mathbf{w}) \mathbf{w} R(\mathbf{w})$ is in $\mathcal{P}$. By symmetry it suffices to show that Left loses if she moves first. Clearly the move from $L(\mathbf{w}) \mathbf{w} R(\mathbf{w})$ to $\mathbf{w} R(\mathbf{w})$ is bad. But a move to $a \mathbf{w} R(\mathbf{w})$ is equally bad, since $a \mathbf{w}$, being of even length is not in $\mathcal{N}$, hence is $\mathcal{P}$ or $\mathcal{R}$ (since $a<L(\mathbf{w})$ ) and again Right wins by removing his entire last heap.

From any point below and leftwards of $L(\mathbf{w}) \mathbf{w} R(\mathbf{w})$, the first player wins by dropping his or her end heap. If there are no such points then the position at hand is in the first row or first column, and any $\mathcal{P}$ so situated is always the triple point of its phase diagram.

For $\mathbf{w}$ of even length the situation is complicated by the fact that $\mathbf{w} \in \mathcal{L}$ does not imply that $L(\mathbf{w})=1$, since Right may have a move which drops a heap from $\mathbf{w}$ and leaves an $\mathcal{N}$ position. However we do know that the leftmost-bottommost $\mathcal{P}$ in the phase diagram of $\mathbf{w}$ is always in the first row or first column, and so it is:

$$
\begin{array}{cl}
1 \mathbf{w} R(\mathbf{w}) & \text { if } \mathbf{w} \in \mathcal{L}, \\
L(\mathbf{w}) \mathbf{w} 1 & \text { if } \mathbf{w} \in \mathcal{R}, \\
1 \mathbf{w} 1 & \text { if } \mathbf{w} \in \mathcal{P} .
\end{array}
$$

To see this, suppose that there is a $\mathcal{P}$ in position $s>1$ of the first row of the phase diagram for $\mathbf{w}$. That means that $\mathbf{w} \in \mathcal{L}$ and $1 \mathbf{w} s \in \mathcal{P}$. Since $\mathbf{w} \in \mathcal{L}$, Right's winning option as second player from $1 \mathbf{w} s$ (first from $\mathbf{w} s$ ) must be to $\mathbf{w}(s-1)$, that is, $\mathbf{w}(s-1) \in$ $\{\mathcal{R}, \mathcal{P}\}$. But if Right moves first from $1 \mathbf{w} s$ to $1 \mathbf{w}(s-1)$ then Left's only reply is to $\mathbf{w}(s-1)$ so this position is in $\mathcal{L}$ or $\mathcal{P}$. Together these conditions imply that $\mathbf{w}(s-1) \in \mathcal{P}$. Therefore $s-1=R(\mathbf{w})-1$, and so $s=R(\mathbf{w})$.

These remarks establish a recursive procedure for determining the type of a position. For if we have a position $a b c . . x y z$ then its type is known if we know the phase diagram of $b c \ldots x y$. To know the phase diagram of $b c \ldots x y$ we need to know its type, and then either $L(b c \ldots x y)$ or $R(b c \ldots x y)$ or possibly both. Its type is recursively determined, and $L(b c \ldots x y)$ is determined from the phase diagram of $b c \ldots x$, while $R(b c \ldots x y)$ is determined from the phase diagram of $c \ldots x y$.

The bases of the recursion are the phase diagrams for positions of length 1 or 2 whose types and triple points are:

\begin{tabular}{ccc} 
Position & Type & Triple Point \\
\hline$a$ & $\mathcal{N}$ & $(a+1) a(a+1)$ \\
$a a$ & $\mathcal{P}$ & $1 a a 1$ \\
$a b(a>b)$ & $\mathcal{L}$ & $1 a b(a+1)$ \\
$a b(b>a)$ & $\mathcal{R}$ & $(b+1) a b(1)$.
\end{tabular}

Actually, this procedure can be tedious. To provide a more efficient means of characterizing the type of a Partizan End Nim position we introduce a new sort of threshold function.

$$
R^{*}(\mathbf{w})=\min \{s: s>0 \text { and } \mathbf{w} s \in\{\mathcal{R}, \mathcal{N}\}\}
$$

In other words, $R^{*}(\mathbf{w})$ is the least positive integer which when appended to $\mathbf{w}$ results in a game which is a win for Right if he moves first. Of course $L^{*}$ is defined similarly. Given 
a position $a \mathbf{w} b$ we can work out its type according to the relationships between $a, b$ and the values of $L^{*}(\mathbf{w} b)$ and $R^{*}(a \mathbf{w})$ according to the following obvious rules:

$$
a \mathbf{w} b \in \begin{cases}\mathcal{L} & \text { if } a \geq L^{*}(\mathbf{w} b) \text { and } b<R^{*}(a \mathbf{w}) \\ \mathcal{R} & \text { if } a<L^{*}(\mathbf{w} b) \text { and } b \geq R^{*}(a \mathbf{w}) \\ \mathcal{N} & \text { if } a \geq L^{*}(\mathbf{w} b) \text { and } b \geq R^{*}(a \mathbf{w}) \\ \mathcal{P} & \text { if } a<L^{*}(\mathbf{w} b) \text { and } b<R^{*}(a \mathbf{w})\end{cases}
$$

The advantage of using the functions $L^{*}$ and $R^{*}$ as opposed to $L$ and $R$ is that we can give simple rules for their computation. Namely:

Proposition 8 The function $R^{*}$ satisfies the following relationships for all positive integers $a$ and strings $\mathbf{w}$.

- If $\mathbf{w} \in\{\mathcal{L}, \mathcal{P}\}$ then

$$
R^{*}(a \mathbf{w})=a+R^{*}(\mathbf{w})
$$

- If $\mathbf{w} \in \mathcal{N}$ then

$$
R^{*}(a \mathbf{w})= \begin{cases}1 & \text { if } a<L^{*}(\mathbf{w}), \\ a-L^{*}(\mathbf{w})+R^{*}(\mathbf{w})+1 & \text { if } a \geq L^{*}(\mathbf{w}) .\end{cases}
$$

- If $\mathbf{w} \in \mathcal{R}$ then

$$
R^{*}(a \mathbf{w})=1+\max \left(a+1-L^{*}(\mathbf{w}), 0\right)
$$

Dual rules apply to the function $L^{*}$. Also $L^{*}(a)=R^{*}(a)=a+1$, and this together with the rules above suffices to determine $L^{*}$ and $R^{*}$.

Proof: We must always be able to show that Right wins as first player in the game $a \mathbf{w} b$ where $b$ is the proposed value for $R^{*}(a \mathbf{w})$ but fails to do so in the game $a \mathbf{w}(b-1)$ (the latter step being unnecessary if $b=1$ !).

Suppose that $\mathbf{w} \in\{\mathcal{L}, \mathcal{P}\}$, and let $b=a+R^{*}(\mathbf{w})$. Right begins in $a \mathbf{w} b$ by removing a single box from his stack. If Left responds by removing her entire stack then since $b-1 \geq R^{*}(\mathbf{w})$, Right will win in the remaining game. As long as Left continues to remove single boxes, Right will do likewise, and at the moment when Left clears her end, Right will still be in a winning position. On the other hand in the game $a \mathbf{w}(b-1)$, Right cannot afford to remove his entire stack as a first move, for Left would do likewise, leaving w which Left as second player will win. So Right will try to win by removing a single box. Then Left will again do likewise, and this time, at the moment when Left clears her end, the remaining position will be $\mathbf{w}\left(R^{*}(\mathbf{w})-1\right)$ which Right cannot win as first player, by the very definition of $R^{*}$.

If $\mathbf{w} \in \mathcal{N}$ and $a<L^{*}(\mathbf{w})$ then Right has a good first move from $a \mathbf{w} 1$ to $a \mathbf{w}$. So in this case $R^{*}(a \mathbf{w})=1$. If $a \geq L^{*}(\mathbf{w})$, then in the phase diagram of $\mathbf{w}$ the row $a \mathbf{w}$ is on or above the line containing the triple point of $\mathbf{w}$. The first $\mathcal{P}$ in this line occurs at position $a-L(\mathbf{w})+R(\mathbf{w})$ and so the first $\mathcal{R}$ occurs at $a-L(\mathbf{w})+R(\mathbf{w})+1$. However, as $\mathbf{w} \in \mathcal{N}$ it is easy to see that $L(\mathbf{w})=L^{*}(\mathbf{w})$ and $R(\mathbf{w})=R^{*}(\mathbf{w})$ since positions $x \mathbf{w}$ and $\mathbf{w} y$ are of 
even length, so never of type $\mathcal{N}$, and thus the winning conditions defining the $*$ functions and their unstarred counterparts are the same. So in this case:

$$
R^{*}(a \mathbf{w})=a-L^{*}(\mathbf{w})+R^{*}(\mathbf{w})+1
$$

as claimed.

Finally, if $\mathbf{w} \in \mathcal{R}$ and $a<L^{*}(\mathbf{w})$ then Right wins as first player in $a \mathbf{w} 1$. For $a \geq L^{*}(\mathbf{w})$ set

$$
b=a+2-L^{*}(\mathbf{w})
$$

and consider the play in $a \mathbf{w} b$ with Right moving first. As usual he begins by removing a single box. If Left ever responds by removing her stack, Right will do the same and win easily since $\mathbf{w} \in \mathcal{R}$. So Left will attempt to delay matters by removing a single box at a time. The value of $b$ ensures that when Right finally clears his stack, Left will have reduced hers to a value less than $L^{*}(\mathbf{w})$, and so she will not win the remaining game. However with even one box fewer in his end stack, Right will not be able to ensure this, and in fact when he clears his end stack, the Left end will equal or exceed $L^{*}(\mathbf{w})$ and so Left will win the remaining game.

The other claims in the proposition are obvious.

That concludes our analysis of Partizan End-Nim. Oh yes, the left and right thresholds of 35551 are 14 and 18 , while those of 35451 are 1 and $3(35551 \in \mathcal{N}$ and $35451 \in \mathcal{L})$. Such peculiar behavior is by no means uncommon (this example was chosen from a random list of 20 length 5 positions). The two $\mathcal{L}$ positions 15332 and 15232 have right thresholds of 11 and 2 respectively.

\section{Remarks, conclusions, and assorted ramblings}

Already in [2] a number of variations of the game are proposed. To us, the most natural (for the impartial version) seems to be the following. The base positions are trees, each vertex carrying a label which is a positive integer. A move is to reduce the label on any leaf. When such a label is reduced to 0 , the leaf is deleted. We have no idea how to play this game in general.

An alternative type of accessibility rule would be to begin with any graph, with one or more depot vertices labelled 0 . Other vertices carry positive integer labels. A vertex is accessible, that is, its label can be reduced, if (and only if) it is adjacent to a vertex which carries a 0 label. Of course, when the label of a vertex is reduced to 0 , that allows access to its previously inaccessible neighbours. For example, a rectangular grid, with its boundary vertices all labelled 0 , corresponds to the fork-lift problem where the stacks of boxes are tightly packed into a grid (but removing a stack of boxes makes all the adjacent stacks available). Partizan versions of this situation can also be imagined, if for example, the drivers are constrained to travel to a non-empty stack from a home position, along a sequence of edges carrying a particular colour. For example, ordinary Partizan End-Nim corresponds to playing on a cycle, with the edges at the common home vertex coloured 
red and blue respectively, and all other edges being colored green (accessible to either player).

The analysis of End-Nim was made easier by a simple Mathematica notebook, written to analyze positions. Any symbolic computation language would have done as well, and for small scale projects it is a procedure that we recommend. For a larger project, using special purpose software such as the Gamesman's Toolkit [3] would be more suitable. In fact we have used this Toolkit to consider the canonical forms of Partizan End-Nim positions. For positions with three heaps or fewer it is relatively straightforward to find these canonical forms, and to justify the observed patterns inductively. However, once positions arise with four or more heaps, the canonical forms are not at all transparent.

The authors would like to thank the anonymous referees for their helpful comments and suggestions, including some which curtailed our more extravagant rhetorical flourishes. The first author would like to acknowledge the stimulating environment of the New Zealand Mathematics Research Institute workshop in Kaikoura, January 2000, where he reacquainted himself with combinatorial games. The second author would like to acknowledge the hospitality of the Department of Mathematics and Statistics at Otago University, and the support of the Beverley Trust.

\section{References}

[1] E.R. Berlekamp, J.H. Conway, and R.K. Guy, Winning Ways, For Your Mathematical Plays, Vol 1, Second edition, AKPeters Ltd., 2001.

[2] Richard K. Guy, Unsolved problems in combinatorial games, in: Games of No Chance, ed. Richard J. Nowakowski, Cambridge University Press, 1998. pp. 475-491

[3] David Wolfe, The Gamesman's Toolkit, in: Games of No Chance, ed. Richard J. Nowakowski, Cambridge University Press, 1998. pp. 93-98 